\title{
Factores asociados a la lactancia materna exclusiva
}

\author{
ROSA NIÑO M. ${ }^{1}$, GIOCONDA SILVA E. ${ }^{2}$, EDUARDO ATALAH S. ${ }^{3}$ \\ 1. Matrona. Escuela de Obstetricia. Facultad de Medicina. Universidad de Chile. \\ 2. Matrona. Escuela de Obstetricia. Facultad de Medicina. Universidad de Chile. \\ 3. Médico. Departamento de Nutrición. Facultad de Medicina. Universidad de Chile.
}

\begin{abstract}
Determinants of exclusive breastfeeding in health centers in Santiago, Chile

Introduction: Limited information is available regarding factors contributing to exclusive breastfeeding until the sixth month. This information is needed to design appropriate policy interventions. Methods: A survey about socio-demographic characteristics, information received, onset and duration of breastfeeding and causes of weaning was applied. Factors associated with exclusive breastfeeding for 6 months were determined by multivariate logistic regression. Results: 256 mothers attending a public sector hospital and 158 mothers attending a private sector hospital responded to the survey. The following factors were higher in the private sector group: Education level, primiparity, cesarean sections and work outside the home $(\mathrm{p}<0.02) .45 .8 \%$ of mothers continued exclusive breastfeeding up to 6 months, with no difference seen between groups. The main causes of weaning were: maternal decision $(27.2 \%)$, perceived hunger $(24.1 \%)$, esthetic factors $(17.3 \%)$ and child's illness (13.0\%). Exclusive breastfeeding up to 6 months was associated with a previous successful breastfeeding (OR 5.4, 95\% CI 2.2-13.2), female child (OR 5.5 CI 2.5-12.3), lower maternal education (OR 5.4 CI 2.4-11.7) and public health system (OR 2.1 CI 1.0-4.5). Conclusion: Weaning is often associated with maternal perceptions rather than objective data. Socio-demographic variables influence successful prolonged breastfeeding. Better education in health factors and breastfeeding might improve this situation.
\end{abstract}

(Key words: Breastfeeding, weaning, nutrition, feeding, chilhood).

Rev Chil Pediatr 2012; 83 (2): 161-169

\section{RESUMEN}

Introducción: Existe limitada información de los factores que contribuyen a una lactancia materna exclusiva hasta el sexto mes, datos necesarios para diseñar adecuadas políticas de intervención. Pacientes y Método: Estudio de corte transversal. Se aplicó una encuesta sobre características socio-demográficas, información recibida, inicio y duración de la lactancia y causas de destete. Por regresiones logísticas multivariadas se determinaron factores asociados a lactancia exclusiva de 6 meses. Resultados: 256 madres del sector público y 158 del sector privado fueron incluidas. Se observó mayor escolaridad, primiparidad, cesáreas y trabajo fuera del hogar en sistema privado $(p<0,02) ; 45,8 \%$ de madres mantuvo lactancia exclusiva hasta 6 meses, sin dife-

Trabajo recibido el 21 de septiembre de 2011, devuelto para corregir el 14 de noviembre de 2011, segunda versión 20 de diciembre de 2011, aceptado para publicación el 13 de enero de 2012.

Correspondencia a:

Eduardo Atalah S.

E-mail: eatalah@med.uchile.cl 
rencias entre grupos. Principales causas de destete: decisión materna $(27,2 \%)$, percepción de hambre $(24,1 \%)$, factores estéticos $(17,3 \%)$ y enfermedad del niño (13,0\%). La lactancia exclusiva hasta los 6 meses se asoció con una lactancia previa exitosa (OR 5,4, 95\% IC 2,2-13,2) sexo femenino del hijo (OR 5,5, IC 2,5-12,3), menor escolaridad materna (OR 5,4, IC 2,4-11,7) y sistema público de salud (OR 2,1, IC 1,0-4,5). Conclusión: El destete muchas veces está relacionado con percepciones maternas más que con datos objetivos. Variables socio-demográficas influyen en lactancia exitosa. Mayor educación en controles de salud y clínicas de lactancia mejorarían esta situación.

(Palabras clave: Lactancia, destete, nutricion, alimentación, lactante).

Rev Chil Pediatr 2012; 83 (2): 161-169

\section{Introducción}

Una lactancia hasta el sexto mes de vida, favorece un adecuado crecimiento y desarro$1 \mathrm{o}^{1}$, impactando en causas evitables de morbi-mortalidad infantil, sin distinción de nivel socioeconómico o trabajo materno ${ }^{2}$. Un mejor conocimiento de los beneficios de la LM, está asociado a inicio temprano y mayor duración de Lactancia materna (LM) ${ }^{3,4}$. La educación en salud, las intenciones prenatales, experiencias de amamantamiento y el apoyo a la madre, inciden en el inicio o suspensión temprana de la $\mathrm{LM}^{5-8}$.

La OMS sugiere "mantener como patrón fundamental la lactancia materna exclusiva (LME) hasta los seis meses y luego, junto con otros alimentos, hasta los dos años de vida", pero hay países lejanos a esta meta ${ }^{9}$. Chile logró aumentar la LME al sexto mes de $16 \%$ en 1993 a 43\% el 2002 en el sector público, según encuestas nacionales ${ }^{10}$. En forma más reciente, las estadísticas regulares del MINSAL muestran un descenso de la prevalencia de LME al sexto mes de vida de 49,0 a 43,5\% entre 2008 y 2010.

Para revertir esta situación se ha realizado una reedición actualizada del Manual de Fomento de Lactancia Materna del MINSAL ${ }^{11}$ y existe un proyecto de ley para prolongar hasta los 6 meses el descanso post natal, que está en trámite en el Congreso. Sin embargo, según MINSAL, la prevalencia de LME al primer mes de vida es $78 \%$ y al tercer mes $66 \%$. Por lo cual, antes del tercer mes de vida la tasa de deserción afecta a un tercio de los niños, siendo un importante desafío de salud pública para el país.
Existe muy limitada información nacional sobre las causas de destete precoz, lo que impide definir las intervenciones que pudieran ser más costo-efectivas. Estudios en diversos países han identificado que la duración de la lactancia materna exclusiva se relaciona fundamentalmente con la edad materna, paridad, nivel socioeconómico, tabaquismo, consejería en lactancia, uso de chupete, jornada laboral y algunas características del recién nacido ${ }^{12-17}$. Un estudio reciente en Chile demostró que el uso de sueros o de sustitutos de la lactancia materna en la maternidad reduce la probabilidad de tener una lactancia materna exclusiva al mes de vida ${ }^{18}$.

Casi no existe información sobre prácticas de LM en el sistema privado, el que representa un tercio de la población del país. En ese escenario, cabe preguntarse además ¿Cuáles son actualmente, los factores de destete precoz en Chile?. Con este propósito se realizó este estudio exploratorio para conocer la prevalencia de lactancia materna y causas de destete precoz, en mujeres de distinto nivel socioeconómico controladas en el sistema público y privado de salud de Santiago.

\section{Pacientes y Método}

\section{Diseño}

Estudio descriptivo transversal en una muestra de mujeres atendidas en el sistema público y privado de atención de salud en Santiago de Chile.

\section{Tamaño de muestra}

Se estimó una diferencia de 12 puntos porcentuales en la prevalencia de lactancia 
materna exclusiva entre el sistema público y privado de atención de salud al sexto mes, nivel de confianza 0,05 , poder 80 y un test de una cola, lo que definió un tamaño de muestra de 220 mujeres en cada grupo. Aún cuando no existe información adecuada para estimar la prevalencia de lactancia materna en el sistema privado de salud, arbitrariamente se consideró que esta sería menor que en el sistema público y la estimación del tamaño muestral se basó en un test de una cola.

\section{Selección de la muestra}

Las madres se seleccionaron en las salas de espera de dos consultorios del sistema público y dos del sistema privado de atención de salud, cuando asistían al control de crecimiento y desarrollo de sus hijos/hijas, durante los meses de octubre y noviembre de 2010.

Criterios de inclusión: Madres entre 3 y 8 meses post parto, con antecedentes obstétricos de parto o cesárea, que aceptaron participar y firmar el consentimiento informado.

Criterios de exclusión: Madres con post parto con patología sistémica grave o enfermedad grave del hijo/a.

\section{Instrumento}

Fue desarrollado en el marco de lo mencionado por Hernández, Fernández y Baptista ${ }^{19}$, como una encuesta de respuestas cerradas y codificadas. Fue sometido a una validez de contenido a través de una consulta de revisión de expertos, quienes ajustaron enunciados de concepto en referencia a conocimientos, actitudes y prácticas.

La capacidad/facilidad se evaluó para entender la idea de las preguntas y se aplicó la medida de estabilidad del instrumento, mediante un muestreo no probabilístico por conveniencia de 30 madres (previo consentimiento informado), aplicando la confiabilidad de Test-Retest.

Posteriormente, fue aplicado a las madres en las instituciones de salud pública y privada seleccionadas, por profesionales de la salud y estudiantes del último año de la Escuela Obstetricia, Facultad de Medicina de la Universidad de Chile. Se resguardaron los aspectos éticos recomendados internacionalmente, otorgando la debida información a las madres encuestadas, así como también respetando su autonomía, y confidencialidad, anexando la firma de un consentimiento informado a cada una de las participantes.

El documento utilizado posee un total de 20 ítems agrupados en las siguientes áreas (anexo 1):

- Variables sociodemográficas maternas: edad, paridad, nivel educativo, ocupación, estado civil.

- Antecedentes del recién nacido: vía del parto, sexo, peso al nacer.

- Variables sociodependientes, que evalúan características intervinientes en la duración de la lactancia materna: experiencia previa en LM anteriores, orientaciones recibidas sobre ventajas e importancia de la LM, momento en que recibió la información, principales fuentes de información, edad de inicio ingesta de jugos, leche artificial y comidas, causas de abandono de LM.

\section{Análisis estadísticos}

Se generó una base de datos electrónica, que fue procesada con el programa STATA 9.2. En las variables categóricas se utilizaron distribuciones de frecuencia y se comparó la situación entre el sistema público y privado con el test $\chi^{2}$. Por medio de regresiones logísticas de un factor se calculó la razón de disparidad (odds ratio) entre cada una de las variables de exposición estudiadas y la probabilidad de tener una lactancia materna exclusiva hasta los seis meses de edad del niño. Las variables categóricas fueron analizadas en forma dicotómica: edad materna ( $<25$ años vs 25 años y más), escolaridad (universitaria $v s$ básica o media); actividad laboral (trabajadora/estudiante vs dueña de casa), vía de parto (cesárea vs parto vaginal), estada civil (casada vs soltera/conviviente), información sobre la lactancia (sí vs no), sistema de salud (público vs privado), paridad (primigesta vs multípara) y experiencias anteriores de lactancia (exitosa vs sin experiencia o regular/ mala). En una segunda etapa se realizó un análisis de regresión logística multifactorial con el procedimiento de adición de variables una a una (forward stepwise), considerando como criterio de inclusión una probabilidad de 0,05 y 
de eliminación de 0,2 . Los modelos estadísticos propuestos permiten estudiar el efecto conjunto de exposición a distintos factores, controlando el efecto de variables de confusión. En todos los casos se consideraron significativos valores de $\mathrm{p}<0,05$.

\section{Resultados}

Se realizaron 414 encuestas (256 sector público y 158 sector privado), eliminándose 3 por datos incompletos. Las madres tenían edad promedio de $28,2 \pm 5,9$ años y sus carac- terísticas sociodemográficas se presentan en la tabla 1. La mayor proporción tenía enseñanza media, con un mejor nivel de escolaridad en el sector privado $(\mathrm{p}<0,001)$. También hubo diferencias en el número de hijos, con mayor proporción de primíparas en el sector privado $(\mathrm{p}<0,001)$. Hubo una proporción similar de madres casadas, convivientes y solteras, sin diferencias entre ambos grupos. La mayor parte de las madres encuestadas trabajaba fuera del hogar, con mayor proporción en el grupo del sistema privado $(\mathrm{p}<0,01)$. Destaca también que el $10 \%$ de las madres eran estudiantes. Casi la mitad de los partos fue por cesárea, con

Tabla 1. Características sociodemográficas de las madres según sistema de salud $(n=411)$

\begin{tabular}{|c|c|c|c|c|c|c|c|}
\hline \multirow{2}{*}{$\begin{array}{l}\text { Variable } \\
\text { Edad años }\end{array}$} & \multicolumn{2}{|c|}{ Sector Público } & \multicolumn{2}{|c|}{ Sector Privado } & \multicolumn{2}{|c|}{ Total } & \multirow[t]{2}{*}{$\chi^{2} \mathbf{p}$} \\
\hline & $\mathrm{n}$ & $\%$ & $\mathrm{n}$ & $\%$ & $\mathrm{n}$ & $\%$ & \\
\hline 16 a 24 & 82 & 32,4 & 45 & 28,5 & 127 & 30,9 & \\
\hline 25 a 29 & 69 & 27,3 & 49 & 31,0 & 118 & 28,7 & \\
\hline 30 a 34 & 65 & 25,7 & 36 & 22,8 & 101 & 24,6 & \\
\hline 35 a 44 & 37 & 14,6 & 28 & 17,7 & 65 & 15,8 & \\
\hline Total & 253 & 100,0 & 158 & 100,0 & 411 & 100,0 & NS \\
\hline \multicolumn{8}{|l|}{ Escolaridad } \\
\hline Básica & 45 & 17,8 & 8 & 5,1 & 53 & 12,9 & \\
\hline Media & 151 & 59,7 & 60 & 37,9 & 211 & 51,3 & \\
\hline Universitaria & 57 & 22,5 & 90 & 57,0 & 147 & 35,8 & \\
\hline Total & 253 & 100,0 & 158 & 100,0 & 411 & 100,0 & $<0,001$ \\
\hline \multicolumn{8}{|l|}{ Paridad } \\
\hline Primípara & 94 & 37,2 & 94 & 59,5 & 188 & 45,7 & \\
\hline Multípara & 159 & 62,8 & 64 & 40,5 & 223 & 54,3 & \\
\hline Total & 253 & 100,0 & 158 & 100,0 & 411 & 100,0 & $<0,001$ \\
\hline \multicolumn{8}{|l|}{ Estado Civil } \\
\hline Soltera & 66 & 26,1 & 49 & 31,0 & 115 & 28,0 & \\
\hline Casada & 85 & 33,6 & 57 & 36,1 & 142 & 34,5 & \\
\hline Conviviente & 102 & 40,3 & 52 & 32,9 & 154 & 37,5 & \\
\hline Total & 253 & 100,0 & 158 & 100,0 & 411 & 100,0 & NS \\
\hline \multicolumn{8}{|l|}{ Ocupación } \\
\hline Ama de Casa & 110 & 43,5 & 34 & 21,5 & 144 & 35,0 & \\
\hline Trabajo fuera hogar & 120 & 47,4 & 105 & 66,5 & 225 & 54,8 & \\
\hline Estudiante & 24 & 9,1 & 19 & 12,0 & 42 & 10,2 & \\
\hline Total & 253 & 100,0 & 158 & 100,0 & 411 & 100,0 & $<0,001$ \\
\hline \multicolumn{8}{|l|}{ Vía del parto } \\
\hline Vaginal & 149 & 58,9 & 61 & 38,6 & 210 & 51,1 & \\
\hline Cesárea & 104 & 41,1 & 97 & 61,4 & 201 & 48,9 & \\
\hline Total & 253 & 100,0 & 158 & 100,0 & 411 & 100,0 & $<0,001$ \\
\hline
\end{tabular}


una proporción significativamente mayor en el sector privado $(61,5$ vs $38,5 \% \mathrm{p}<0,001)$.

El $90,5 \%$ de las encuestadas reconoció haber recibido información de LM (tabla 2). La mayor parte de ellas la recibieron en diferentes períodos, destacando en segundo lugar el rol de los controles prenatales. Llama la atención la baja proporción de madres que reconoce haber recibido información en controles de crecimiento, aunque en muchos casos está incluido en la alternativa "en varios momentos". Destaca también que una proporción mayor de madres no recibió información sobre LM en el sistema privado $(\mathrm{p}<0,02)$. Respecto a la fuente de información casi la mitad de los casos reconoció haberla recibido de diversos integrantes del equipo de salud (médicos, enfermeras, nutricionistas, matronas). En una proporción muy similar, la matrona fue la fuente de información, sin diferencias significativas entre ambos grupos.

La duración de la LME se presenta en la tabla 3, incluyendo sólo a niños que habían cumplido 6 meses (93 del sistema público y

Tabla 2. Información recibida por la madre sobre la lactancia según sistema de salud

\begin{tabular}{|c|c|c|c|c|c|c|c|}
\hline \multirow[t]{2}{*}{ Información sobre lactancia } & \multicolumn{2}{|c|}{ Sector Público } & \multicolumn{2}{|c|}{ Sector Privado } & \multicolumn{2}{|c|}{ Total } & \multirow[t]{2}{*}{$\chi^{2} \mathbf{p}$} \\
\hline & $\mathbf{n}$ & $\%$ & $\mathbf{n}$ & $\%$ & $\mathbf{n}$ & $\%$ & \\
\hline No recibió información & 18 & 7,1 & 21 & 13,3 & 39 & 9,5 & \\
\hline Antes embarazo & 10 & 4,0 & 5 & 3,1 & 15 & 3,6 & \\
\hline Durante embarazo & 65 & 25,7 & 36 & 22,8 & 101 & 24,6 & \\
\hline En la maternidad & 13 & 5,1 & 10 & 6,3 & 23 & 5,6 & \\
\hline Control de crecimiento & 0 & 0,0 & 5 & 3,2 & 5 & 1,2 & \\
\hline Varios momentos & 147 & 58,1 & 81 & 51,3 & 228 & 55,5 & \\
\hline Total & 253 & 100 & 158 & 100 & 411 & 100 & $<0,02$ \\
\hline \multicolumn{8}{|l|}{ Fuente de información } \\
\hline Familia & 9 & 3,8 & 9 & 6,6 & 18 & 4,8 & \\
\hline Médico & 11 & 4,7 & 12 & 8,7 & 23 & 6,2 & \\
\hline Enfermera & 5 & 2,1 & 2 & 1,5 & 7 & 1,9 & \\
\hline Matrona & 102 & 43,4 & 49 & 35,8 & 151 & 40,6 & \\
\hline Medios de comunicación & 2 & 0,9 & 1 & 0,7 & 3 & 0,8 & \\
\hline Otras fuentes & 2 & 0,8 & 3 & 2,2 & 5 & 1,3 & \\
\hline Diversos miembros equipo salud & 104 & 44,3 & 61 & 44,5 & 165 & 44,4 & \\
\hline Total & 235 & 100 & 137 & 100 & 372 & 100 & NS \\
\hline
\end{tabular}

Tabla 3. Edad de término de la lactancia materna exclusiva en niños de 6 meses y más según sistema de salud $(n=181)$

\begin{tabular}{|lcccccc|}
\hline Edad / meses & \multicolumn{2}{c}{ Sector Público } & \multicolumn{2}{c}{ Sector Privado } & \multicolumn{2}{c|}{ Total } \\
& $\mathbf{n}$ & $\mathbf{\%}$ & $\mathbf{n}$ & $\mathbf{\%}$ & $\mathbf{n}$ & $\%$ \\
\hline Menos de 1 & 10 & 10,8 & 18 & 20,5 & 28 & 15,5 \\
\hline a 2 & 11 & 11,8 & 6 & 6,8 & 17 & 9,4 \\
\hline 3 a 4 & 10 & 10,8 & 5 & 5,7 & 15 & 8,3 \\
\hline 5 & 18 & 19,3 & 20 & 22,7 & 38 & 21,0 \\
\hline 6 y más & 44 & 47,3 & 39 & 44,3 & 83 & 45,8 \\
\hline Total & 93 & 100,0 & 88 & 100,0 & 181 & 100,0 \\
\hline
\end{tabular}


Tabla 4. Causa de abandono de la lactancia materna exclusiva según sistema de salud $(\mathbf{n}=162)$

\begin{tabular}{|c|c|c|c|c|c|c|}
\hline \multirow[t]{2}{*}{ Motivo de abandono } & \multicolumn{2}{|c|}{ Sector Público } & \multicolumn{2}{|c|}{ Sector Privado } & \multicolumn{2}{|c|}{ Total } \\
\hline & $\mathbf{n}$ & $\%$ & $\mathbf{n}$ & $\%$ & $\mathbf{n}$ & $\%$ \\
\hline Enfermedad del niño & 7 & 8,4 & 14 & 17,7 & 21 & 13,0 \\
\hline Enfermedad de la madre & 1 & 1,2 & 1 & 1,3 & 2 & 1,2 \\
\hline Niño rechaza pecho & 2 & 2,4 & 5 & 6,3 & 7 & 4,3 \\
\hline Queda con hambre & 30 & 36,2 & 9 & 11,4 & 39 & 24,1 \\
\hline Decisión de la madre & 19 & 22,9 & 25 & 31,6 & 44 & 27,2 \\
\hline Inicio trabajo/estudio & 2 & 2,4 & 10 & 12,7 & 12 & 7,4 \\
\hline Estética & 15 & 18,1 & 13 & 16,5 & 28 & 17,3 \\
\hline Otros & 7 & 8,4 & 2 & 2,5 & 9 & 5,5 \\
\hline Total & 83 & 100,0 & 79 & 100,0 & 162 & 100,0 \\
\hline
\end{tabular}

$\chi^{2}=23,91 ; p<0,001$

Tabla 5. Factores asociados a una lactancia materna exclusiva de 6 meses según modelo de regresión logística multivariado

\begin{tabular}{|llc|}
\hline Variable & OR & 95\% IC \\
\hline Experiencia exitosa en una lactancia previa & 5,4 & $2,2-13,2$ \\
\hline Sexo femenino del recién nacido & 5,5 & $2,5-12,3$ \\
\hline Escolaridad materna básica o media & 5,4 & $2,4-11,7$ \\
\hline Sector publico de salud & 2,1 & $1,0-0,4,5<$ \\
\hline
\end{tabular}

Pseudo $\chi^{2} 0,23 p<0,001$.

88 del sistema privado). Destaca casi un 25\% de las madres suspendió la LME antes de tres meses y el $46 \%$ la mantuvo hasta los 6 meses, proporción similar a la información del MINSAL. Aunque hubo destete más precoz en el sistema privado, las diferencias no fueron significativas (p NS).

Según lo referido por las madres los principales motivos de abandono de la lactancia fueron "decisión propia" y percepción materna que el niño "quedaba con hambre" (tabla 4), con comportamiento diferente entre ambos grupos $(\mathrm{p}<0,001)$. En una proporción significativa el destete se vinculó a problemas de estética y en menor frecuencia a la salud del niño, trabajo o estudio materno. En la mayor parte de estas variables el comportamiento fue diferente entre las beneficiarias de ambos servicios de salud $(\mathrm{p}<0,001)$.

El análisis por regresión logística univariada no mostró una asociación significativa entre una lactancia materna hasta los 6 meses y la edad de la madre, paridad, estado civil, información sobre la lactancia, momento que la recibió y sistema de salud. Las variables asociadas a una lactancia exitosa fueron una experiencia previa positiva (OR 3,0 95\% IC 1,5-62), una menor escolaridad materna (OR 4,7 95\% IC 2,4-9,2), ser dueña de casa (OR $1,895 \%$ IC $1,0-2,9)$ y sexo femenino del lactante (OR 3,6 95\% IC 1,9-7,0). El análisis multivariado excluyó la variable actividad laboral y consideró el sistema de salud, aunque en el límite de la significación estadística (tabla 5). Estas cuatro variables explicaron el 23\% de la varianza respecto a duración de la lactancia materna exclusiva al sexto mes.

\section{Discusión}

Existen muy pocos datos nacionales sobre las causas del destete precoz, a pesar de la amplia preocupación que ha habido sobre el tema en los últimos 20 años. Tampoco existe 
información sobre la situación de lactancia el sistema privado de salud, la que representa un tercio de la población nacional. Este es el principal aporte de este estudio, que nos demuestra que casi en la mitad de los casos el destete precoz está asociado a decisiones o percepciones maternas, que no necesariamente reflejan la realidad. La impresión de que el niño "queda con hambre" no siempre está asociada a un hecho objetivo o no se relaciona con la curva de crecimiento del niño. Ello podría reducirse con una mayor información en los controles de salud o con clínicas de lactancia materna. Preocupa también la alta proporción de casos en el que el destete está asociado a razones estéticas, a pesar de las insuperables ventajas de la lactancia materna, lo que refleja probablemente que la información recibida no ha sido suficiente o adecuada.

El trabajo o estudio materno aparece en una muy baja proporción como causa de destete, lo que puede poner en duda el potencial impacto de la ley de prolongación del descanso postnatal. Sin embargo, es posible que pudiera haber algún grado de superposición con la respuesta "decisión materna", ya que las madres que deben incorporarse en las próximas semanas a un actividad laboral, puede que se "preparen previamente" iniciando una alimentación complementaria.

Otro dato interesante de destacar en la información recibida sobre lactancia materna. Aunque el 90\% de las madres recibió información sobre la importancia de la lactancia materna, no es menor el hecho que eso no ocurrió en el $10 \%$ restante, proporción que fue significativamente mayor en el sistema privado de salud. Algo más de la mitad de las madres recibió información en varios momentos y de distintas fuentes, aunque lo ideal sería que eso ocuriera en todos los casos, lo que refuerza la necesidad de enfatizar el tema durante el embarazo, parto, puerperio y controles de crecimiento y desarrollo.

La prevalencia de lactancia materna exclusiva a los 6 meses mostró cifras parecidas a la información que genera el Ministerio de
Salud a través del Departamento de Estadísticas e Información en Salud a nivel nacional, lo que contribuye a validar esta información. Con alguna sorpresa la situación fue similar en el sistema privado, lo que sugiere un avance muy importante en este grupo de población, aunque deben tenerse en cuenta las limitaciones de este estudio.

Se observó que a menor edad materna disminuye el período de lactancia, grupo que debiera ser prioritario para el equipo de salud en las acciones de promoción y prevención ${ }^{20}$. La alta incidencia de cesáreas es un problema a resolver, para que no afecte el inicio precoz del amamantamiento que favorece la LME hasta los 6 meses. Dos de los factores asociados en este estudio a una lactancia exclusiva hasta los 6 meses (experiencia previa exitosa, menor nivel de escolaridad) han sido descritos en la literatura ${ }^{13,15,16}$. Sin embargo, llama la atención la importante diferencia en función del sexo del lactante, lo que no ha sido descrito previamente. Este hecho sugiere la necesidad de evaluar esta asociación en estudios de mayor representatividad. Aún cuando el sistema de salud no demostró diferencias en los análisis univariados, si se demostró una situación más favorable en el sistema público, al utilizar modelos multivariados.

En la interpretación de resultados se deben considerar algunas limitantes del estudio, como su reducido tamaño de muestra, uso de una muestra no aleatoria y que las madres del sistema privado de salud, correspondían a un nivel socioeconómico más bien medio y no alto. Los datos se obtuvieron a través de una encuesta realizada en algunos casos varios meses después del destete, lo que podría determinar menor confiabilidad de la información por una sobreestimación de la duración real de la lactancia ${ }^{21}$.

A pesar de ello se aportan datos de interés para proponer y fortalecer futuras intervenciones, con acciones coordinadas del equipo de salud, la mujer y su familia durante el embarazo, parto y puerperio, en todas las instancias de contacto y seguimiento ${ }^{22}$. 
R. NIÑO M. y cols.

Anexo 1. Encuesta lactancia materna

\begin{tabular}{|c|c|c|c|}
\hline Ítem & Variable & Alternativas de respuesta & Código \\
\hline 1 & Centro de Salud & Público $=1 ;$ Privado $=2$ & \\
\hline 2 & Número encuesta & & \\
\hline 3 & Edad de la madre & Años & \\
\hline 4 & Escolaridad materna & $\begin{array}{l}\text { Básica incompleta }=1 \text {, Básica completa }=2 \text {, Media incom- } \\
\text { pleta }=3 \text {, Media completa }=4 \text {, Universitaria incompleta }= \\
5, \text { Universitaria completa }=6\end{array}$ & \\
\hline 5 & Ocupación & Ama de casa $=1$, Trabajadora $=2$, Estudiante $=3$ & \\
\hline 6 & Estado Civil & Soltera $=1$, Casada $=2$, Conviviente $=3$, Sin pareja $=4$ & \\
\hline 7 & Número total de hijos & Registre sólo recién nacidos vivos & \\
\hline 8 & $\begin{array}{l}\text { Orientaciones previas sobre ventajas e impor- } \\
\text { tancia LM }\end{array}$ & $\mathrm{Si}=1, \mathrm{No}=2$ & \\
\hline 9 & ¿Cuándo las recibió? & $\begin{array}{l}\text { Antes del embarazo }=1 \text {, Durante el embarazo }=2 \text {, Des- } \\
\text { pués del parto en la maternidad }=3 \text {, Después del parto en } \\
\text { controles de salud }=4 \text {; En varios momentos }=5 \text {. Si se ha } \\
\text { recibido información en varios momentos (Destaque todos } \\
\text { los números que corresponda) }\end{array}$ & \\
\hline 10 & Principales Fuentes de información & $\begin{array}{l}\text { Familia }=1, \text { Médico }=2, \text { Enfermera }=3, \text { Matrona }=4, \\
\text { Medios masivos de comunicación }=5, \text { Amigos } 0 \text { conoci- } \\
\text { dos }=6, \text { Otros }=7 \text {; varios }=8 \text {. (Destaque todos los números } \\
\text { que corresponda) }\end{array}$ & \\
\hline 11 & Fecha nacimiento lactante & $\begin{array}{l}\text { Día } \\
\text { Registre posteriormente la edad actual en meses }\end{array}$ & \\
\hline 12 & Sexo & Femenino $=1 \quad$ Masculino $=2$ & \\
\hline 13 & Vía de nacimiento & Vaginal $=1$ Cesárea $=2$ & \\
\hline 14 & ¿Cuál fue su experiencia en lactancias previas? & $\begin{array}{l}\text { Buena }=1, \text { Regular }=2, \text { Mala }=3, \text { No corresponde, Pri- } \\
\text { mípara }=4\end{array}$ & \\
\hline 15 & $\begin{array}{l}\text { En esta lactancia hasta qué edad le dio sólo } \\
\text { pecho sin ningún otro alimento }\end{array}$ & Edad en meses & \\
\hline 16 & ¿A que edad empezó a darle agua o jugos? & Edad en meses & \\
\hline 17 & ¿A que edad empezó a darle leche artificial? & $\begin{array}{l}\text { Edad en meses. Registre } 9 \text { en caso que aún no recibe fór- } \\
\text { mulas }\end{array}$ & \\
\hline 18 & ¿A que edad empezó a darle comida? & $\begin{array}{l}\text { Edad en meses. Registre } 9 \text { en caso que aún no recibe co- } \\
\text { midas }\end{array}$ & \\
\hline 19 & $\begin{array}{l}\text { ¿Hasta qué edad le dio leche materna además } \\
\text { de otro alimento? }\end{array}$ & $\begin{array}{l}\text { Edad en meses. Registre la edad actual en caso de que aún } \\
\text { recibe pecho }\end{array}$ & \\
\hline 20 & $\begin{array}{l}\text { Principal motivo por el cual abandonó la lac- } \\
\text { tancia materna o sustituyó/ complementó con } \\
\text { leche artificial }\end{array}$ & $\begin{array}{l}\text { Enfermedad del niño }=1 \text {, Enfermedad de la madre con } \\
\text { utilización de fármacos excretados por la leche materna }=2 \text {, } \\
\text { Enfermedad de la madre relacionada con la mama o el pe- } \\
\text { zón }=3 \text {, Rechazo del niño al pecho }=4 \text {, No se llena, queda } \\
\text { con hambre }=5 \text {, Incorporación a la sala cuna }=6 \text {, Falta de } \\
\text { leche }=7 \text {, Voluntariedad de la madre }=8 \text {, Inicio de activida- } \\
\text { des laborales o estudiantiles }=9, \text { Estética }=10 \text {, Ambiente } \\
\text { familiar inadecuado }=11, \text { Otros }=12\end{array}$ & \\
\hline
\end{tabular}




\section{Referencias}

1.- Morán M, Naveiro JC, Blanco Fernández E, Cabañeros I, Rodríguez M, Peral A: Prevalencia y duración de la lactancia materna: influencia en el peso y morbilidad. Nutr Hosp 2009; 24: 213-7.

2.- Bartick M, Reinhold A: The burden of suboptimal breastfeeding in the United States: a pediatric cost analysis. Pediatrics. 2010; 125: e 1048-56.

3.- Kohlhuber M, Rebhan B, Schwegler U, Koletzko B, Fromme $H$ : Breastfeeding rates and duration in Germany. A Bavarian cohort study. Br J Nutr 2008; 99: 1127-32

4.- Sisk PM, Lovelady CA, Gruber KJ, Dillard RG, O'Shea TM: Human milk consumption and full enteral feeding among infants who weigh $\leq 1250$ grams. Pediatrics 2008; 121: e1528-33.

5.- Dyson L, Renfrew MJ, McFadden A, McCormick F, Herbert $G$, Thomas $J$ : Policy and public health recommendations to promote the initiation and duration of breast-feeding in developed country settings. Public Health Nutr 2010; 13: 137-44.

6.- McNiel M, Labbok M, Abrahams S: What are the risks associated with formula feeding? A re-analysis and review. Birth 2010; 37: 50-8.

7.- DiGirolamo A, Thompson $N$, Martorell R, Fein $S$, Grummer-Strawn L: Intention or Experience? Predictors of Continued Breastfeeding Health Educ Behav 2005; 32: 208-26.

8.- Gigena P: Abandono de la lactancia materna exclusiva antes de los 6 meses, en mujeres de Villa el Rosario, Córdoba, Argentina. Claves de Odontología 2008; 15: 41-6.

9.- Merten S, Dratva J, Ackermann-Liebrich U: Do babyfriendy hospitals influence breastfeeding duration on a national level? Peditrics 2005; 116: 702-8.

10.- Comisión Nacional de Lactancia Materna: Efectividad del Programa de Lactancia, Resultados Encuestas Nacionales 1993-2002. Chile, 2005.

11.- Ministerio de Salud: Lactancia Materna: contenidos técnicos para profesionales de la salud. $2^{\circ}$ Edición, Santiago, 2010

12.- França MCT, Giugliani ERJ, Oliveira LD, et al: Uso de mamadeira no primeiro mês de vida: determinantes e influência na técnica de amamentação. Rev Saúde Pública 2008; 42: 607-14.

13.- Baptista GH, Andrade AHKG, Giolo SR: Fatores associados à duração do aleitamento materno em crianças de famílias de baixa renda da região sul da cidade de Curitiba, Paraná, Brasil. Cad Saude Pública 2009; 25 : 596-604.

14.- Franco SC, Nascimento MBR, Reis MAM, Islerr H, Grisi SJFE: Aleitamento materno exclusivo em lactentes atendidos na rede pública do Municipio de Joinville, Santa Catarina, Brasil. Rer Bras Saúde Matern Infant 2008; 8: 291-7.

15.- Barbosa BM, Palma D, Domene SMA, Taddei JAAC, López FA: Fatores de risco associados ao desmame precoce e ao período de desmame em lactentes matriculados em creches. Rev Paul Pediatr 2009; 27: 272-81.

16.- Cabezuelo G, Vidal S, Abeledo A, Frontera P: Factores relacionados con el abandono precoz de la lactancia materna. Rev Esp Pediatr 2006; 62: 212-8.

17.- Villanueva $M$, Álvarez J, Peña L: Factores condicionantes de la lactancia materna durante el puerperio inmediato en el Hospital Universitario Materno-Infantil de Canarias. Rev Española Nutr Comun 2008; 14: 30-41.

18.- Cardoso KI, Toso MP, Valdés LV, Cerda LJ, Manríquez TV, Paiva CG: Introducción Precoz de Sustitutos de Lactancia Materna e Incidencia de Lactancia Materna Exclusiva al Mes de Vida. Rev Chil Pediatr 2010; 81: 326-32.

19.- Hernández R, Fernández C, Baptista P: Metodología de la Investigación. McGraw-Hill Interamericana. México, D. F. Tercera edición 2003; 9: 343-447.

20.- Glass T, Tucker K, Kauffman R, et al: Infant Feeding and Contraceptive Practices Among Adolescents With a High Teen Pregnancy Rate: A 3-Year Retrospective Study Journal of Women's Health 2010; 19: 1659-63.

21.- Cupul-Uicab LA, Gladen BC, Hernández-Ávila M, Longnecker MP: Reliability of reported breastfeeding duration among reproductive-aged women from Mexico. Matern Child Nutr 2009; 5: 125-37.

22.- Lawrence RA, Lawrence RM: Lactancia Materna: una guía para la profesión médica $6^{\mathrm{a}}$ ed. Elsevier Mosby, Madrid, 2007. 